\title{
Rosmarinus officinalis L.: propriedades farmacológicas relacionadas à Odontologia
}

Rosmarinus officinalis L.: pharmacological properties related to Dentistry

Rosmarinus officinalis L.: propiedades farmacológicas relacionadas con la Odontología

\author{
Mírian Alencar de MEDEIROS ${ }^{1}$ \\ José Henrique de Araújo CRUZ ${ }^{1}$ \\ Heloisa Mara Batista Fernandes de OLIVEIRA ${ }^{2}$
}

Gymenna Maria Tenório GUÊNES ${ }^{3}$

Maria Angélica Satyro Gomes ALVES ${ }^{3}$

Abrahão Alves de OLIVEIRA FILHO ${ }^{3}$

${ }^{1}$ Curso de Graduação em Odontologia. Centro de Saúde e Tecnologia Rural, Universidade Federal de Campina Grande, UFCG 58708-110 Patos/PB, Brasil

${ }^{2}$ Farmacêutica em Hospital Universitário Ana Bezerra, Universidade Federal do Rio Grande do Norte, UFRN, 59078-970, Natal/RN, Brasil

${ }^{3}$ Professor(a) Doutor(a) do Curso de Graduação em Odontologia. Centro de Saúde e Tecnologia Rural, Universidade Federal de Campina Grande, UFCG 58708-110 Patos/PB, Brasil

\section{Resumo}

Introdução: Pesquisas na área de fitoterapia têm provado ser um método alternativo e eficaz contra diferentes agravos à condição de saúde humana, por isso, contribuições dela em diferentes áreas, como na Odontologia, tem grande importância como uma alternativa com menos efeitos colaterais comparados aos medicamentos sinteticamente fabricados, uma vez que as plantas medicinais têm demonstrado elevado poder de cura em estado natural. Objetivo: O presente trabalho objetivou apresentar uma revisão de literatura da espécie vegetal R. officinalis L. (Alecrim) em busca por novas possibilidades terapêuticas para a área da saúde, com ênfase na saúde bucal. Metodologia: O estudo trata-se de uma revisão bibliográfica narrativa, realizada no período de 18 de dezembro de 2017 à 28 de maio de 2018, utilizando artigos científicos em língua portuguesa, espanhola e inglesa, retirados das bases de dados Scielo, MedLine, Lilacs e Monografias. Discussão: Evidências científicas têm revelado que o extrato R. officinalis L possui grande poder antibacteriano, antifúngico, antiinflamatório e antioxidante, além de atuar efetivamente com reduções sob biofilmes polimicrobianos para área de Odontologia. Conclusão: Portanto, o efeito antimicrobiano produzido a partir de extratos do R. officinalis $\mathrm{L}$ tem sido positivo segundo relatos da literatura, todavia, mais pesquisas clínicos precisam ser realizados para enriquecer os atuais estudos, já que esta planta possui poder fitoterápico no tratamento de diversas injúrias bucais como gengivite e periodontite causadas como consequência da atividade bacteriana.

Descritores: Fitoterapia; Rosmarinus officinalis; Odontologia.

\section{Abstract}

Introduction: Research in the field of herbal medicine has proven to be an alternative and effective method against different conditions of human health, so its contributions in different areas, such as in dentistry, are of great importance as an alternative with fewer side effects with drugs manufactured synthetically, since medicinal plants have demonstrated high healing power in the natural state. The objective of this work was to present a review of the literature of the plant species R. officinalis L. (Alecrim) in search of new therapeutic possibilities for the health area, with emphasis on oral health. Methodology: The study is a narrative bibliographic review, carried out from December 18, 2017 to May 28, 2018, using scientific articles in Portuguese, Spanish and English, taken from the Scielo, MedLine, Lilacs and Monographs. Discussion: Scientific evidence has revealed that R. officinalis L extract has great anti-bacterial, antifungal, anti-inflammatory and antioxidant properties, in addition to effective reduction with polymicrobial biofilms for the Dentistry area. Conclusion: Therefore, the antimicrobial effect produced from extracts of R. officinalis $\mathrm{L}$ has been positive according to reports in the literature, however, more clinical research needs to be done to enrich the current studies, since this plant has phytotherapeutic power in the treatment of several oral injuries such as gingivitis and periodontitis caused as a consequence of bacterial activity.

Descriptors: Phytotherapy; Rosmarinus officinalis; Dentistry.

\section{Resumen}

La investigación en el campo de la medicina herbácea ha provenido de un método alternativo y eficaz para las distintas condiciones de la salud humana, es su contribución en diferentes áreas, como en odontología, son de gran importancia las alternativas con los efectos secundarios con los efectos secundarios fabricado sintético, debido a las medicinas medicinales han estado en el cura de energía curativa en el estado natural. El objetivo de este trabajo fue presentar una revisión de los casos de la planta de las raíces. R. officinalis L. (Alecrim) en la búsqueda de nuevas terapias terapéuticas para el área de salud, con incidencia sobre la salud oral. Metodología: El estudio es una revisión de la literatura narrativa, realizado a partir de 18 diciembre 2017 a 28 mayo 2018, el uso de artículos científicos en portugués, español e inglés, tomados de la Scielo, MEDLINE y lilas monografías. Que se ha convertido en una de las más importantes de la industria farmacéutica y de la industria farmacéutica. Conclusión: Por lo tanto, el antimicrobiano efecto producido de extractos de R. officinalis L ha sido positivo para informar en los casos, sin embargo, más clínica clínica necesita ser enriquecida en los estudios actuales, desde esta planta ha phytotherapeutic power in the treatment of varios oral oral tales como gingivitis y periodontitis causan la consecuencia de la actividad bacterial.

Descriptores: Fitoterapia; Rosmarinus officinalis; Odontología.

\section{INTRODUÇÃO}

Os estudos sobre as plantas medicinais têm colaborado para espalhar informações sobre sua importância terapêutica, ratificando o conhecimento sobre elas acumulado há séculos, todavia, os componentes químicos dessas plantas medicinais ainda não são totalmente conhecidos e precisam ser examinados ${ }^{1-4}$. Hoje, a maioria das drogas são desenvolvidas a partir de constituintes de plantas medicinais, com base em suas aplicações ${ }^{5,6}$.

A análise da atividade antimicrobiana emerge como um teste para investigar a hipótese da atividade de produtos fitoterápicos para a realização de testes mais precisos com objetivo bactericida ou bacteriostático. Logo, pesquisas que visem o uso de fitofármacos ou produtos medicinais à base de partes de plantas requerem destaque científico, pela capacidade de subsidiar tratamentos de diferentes agravos existentes nas mais diversas áreas da saúde, principalmente Odontologia ${ }^{7}$.

Grande parte das plantas sintetizam metabólitos secundários com ação antimicrobianos, ora pelo crescimento e desenvolvimento, ora em resposta ao estresse ou ataque oriundos do meio ambiente para persistir. Os óleos essenciais (um metabólito secundário) representa uma nova maneira de diminuir a proliferação de microorganismos que 
atacam as plantas, por isso está presente em diversas partes dela ${ }^{8}$.

A $R$. officinalis L. (Lamiaceae) é uma espécie de planta lenhosa perene, advinda do Mediterrâneo, suas folhas são comumente usadas como condimento culinário e também servem para fins medicinais e, na contemporaneidade, pode ser encontrada e cultivada em város continentes como planta ornamental e aromática ${ }^{8}$.

Por ser uma planta que prefere climas mais secos e ensolarados, essas condições climáticas determinarão um melhor desenvolvimento e consequentemente melhor qualidade do óleo essencial produzido por ela ${ }^{9}$. Esta planta também é muito empregada nas populações indígenas, onde cresce naturalmente para usos culinários devido ao aroma característico ${ }^{5}$.

Pesquisas comprovaram que a atividade antimicrobiana in vitro do extrato aquoso e do óleo essencial do Alecrim desempenhou atividade inibitória em cepas de bactérias Gram-negativas sobre culturas, corroborando com os achados na literatura a respeito da sua ação antibacteriana ${ }^{10}$.

Apesar de promissora a prática da medicina alternativa na odontologia, a fitoterapia é ainda pouco explorada, logo, em virtude da busca por novas possibilidades terapêuticas para a área da saúde, com ênfase na saúde bucal, existem poucos estudos que evidenciam a atividade do $R$. officinalis $L$. como fitofármaco no combate aos microrganismos da cavidade oral e as reduzidas opções em produtos disponíveis à base desta planta como forma alternativa às já existente. A partir disso, o presente trabalho objetivou apresentar uma revisão da literatura da espécie vegetal $R$. officinalis $L$. (Alecrim) comum no cotidiano das pessoas e suas propriedades terapêuticas.

MATERIAL E MÉTODO

O estudo trata-se de uma revisão bibliográfica narrativa, que buscar publicações amplas, apropriadas para descrever e discutir o desenvolvimento ou o "estado da arte" de um determinado assunto, sob ponto de vista teórico ou contextual e, dessa forma, na análise da literatura publicada em livros, artigos de revista impressas e/ou eletrônicas na interpretação e avaliação crítica pessoal do autor ${ }^{11}$. A pesquisa foi realizada no período de 18 de dezembro de 2017 à 28 de maio de 2018. Com embasamento para a pesquisa, foram utilizados artigos científicos retirados das seguintes bases de dados: SCIELO (Scientific Eletronic Library Online), MEDLINE (Medical Literature Analysis and Retrieval Sistem Online), Lilacs (Centro Latino-Americano e do Caribe de Informação em Ciências da Saúde), e monografias que atendiam aos requisitos do estudo em questão.

Os artigos utilizados como referências bibliográficas correspondem ao período entre 2014 a 2017, com exceção de artigos clássicos necessários para a escrita do trabalho, do qual a pesquisa foi realizada tanto na língua nacional, português, como em inglês e espanhol. Foram utilizados como descritores isolados, ou combinados, para a busca em base de dados: "Rosmarinnus Officinalis L.", "aspectos botânicos do Rosmarinnus Officinalis L.", "Rosmarinnus officinalis L. usos fitoterápicos", "Rosmarinnus officinalis L. uso na odontologia", para compor a base temática da revisão. Importantes tópicos para discussão foram estudados e organizados da seguinte forma: a) Aspectos Botânicos da Rosmarinus officinalis L.; b) Constituintes Químicos Rosmarinus officinalis L.; c) Constituintes Químicos Rosmarinus officinalis L.; d) Aplicações Odontológicas do Rosmarinus officinalis $L$.

\section{RESULTADOS E DISCUSSÃO}

- Aspectos botânicos da Rosmarinus officinalis $L$

$R$. officinalis $L$. (Lamiaceae) é uma espécie de planta lenhosa perene, oriunda da região do Mediterrâneo, que atualmente pode ser encontrada e cultivada em todos os continentes como planta aromática e ornamental; suas folhas são comumente usadas como condimento culinário e também servem para fins medicinais ${ }^{8}$.

É uma planta que prefere climas mais secos, ensolarados, sendo que estas condições climáticas irão determinar um melhor desenvolvimento e por conseguinte uma melhor qualidade do óleo essencial (metabólito secundário produzido por ela) ${ }^{9}$. Além dos usos culinários devido ao aroma característico, esta planta também é largamente empregada nas populações indígenas, onde cresce naturalmente ${ }^{5}$.

$\mathrm{O}$ uso efetivo de plantas medicinais tem contribuído para disseminar informações sobre sua importância terapêutica e efeitos curativos, validando o conhecimento terapêutico acumulado há séculos, no entanto, os constituintes químicos das plantas medicinais ainda não são totalmente conhecidos e precisam ser investigados ${ }^{1-4}$. Atualmente, a maioria das drogas modernas são desenvolvidas a partir de compostos de plantas medicinais, com base em seus usos e aplicações etnofarmacológicos ${ }^{5,6}$.

Trabalhos que visem à utilização de fitofármacos ou produtos medicinais à base de partes de plantas merecem destaque no âmbito social e científico, pela potencialidade de subsidiar tratamentos de diferentes agravos existentes nas mais diversas áreas da saúde. Avaliar a atividade antimicrobiana surge como teste para verificar a hipótese de ação de produtos fitoterápicos para a realização de testes posteriores mais específicos e precisos com finalidade bactericida ou bacteriostático ${ }^{7}$.

Plantas aromáticas e medicinais podem ser usadas 
para curar doenças humanas, e então compostas naturais derivadas dessas plantas devem ser investigados para obter uma melhor compreensão de suas propriedades, bem como a segurança e eficiência de seu uso clínico-terapêtico ${ }^{12}$.

- Constituintes químicos Rosmarinus officinalis $L$

A maioria das plantas produz metabólitos secundários com ação antimicrobianos, seja pelo seu curso normal de crescimento e desenvolvimento, seja em resposta ao estresse ou ataque de patógenos advindos do meio ambiente como forma de perpetuação. O uso de óleos essenciais (um metabólito secundário) representa uma nova maneira de reduzir a proliferação de microorganismos a que atingem a planta ${ }^{8}$.

$R$. officinalis é composto principalmente de compostos fenólicos, di e triterpenos e óleos essenciais, que por sua vez possuem como características marcante a liberação de odores ${ }^{13,14}$. Seus principais constituintes responsáveis pelas atividades farmacológicas já encontradas, com efeitos, sobretudo, antimicrobianos são: 1,8-cineole $(52,2 \%)$, cânfora $(15,2 \%)$ e $\alpha$-pinene $(12,4 \%)^{15}$.

Os óleos essenciais e os extratos aquosos podem ser utilizados na indústria farmacêutica e para outros fins, de acordo com suas propriedades, além disso, estudos têm demonstrado sua importância no desenvolvimento de novos antimicrobianos e controle de doenças bacterianas ${ }^{16}$.

Para obter os compostos biologicamente ativos do Alecrim, é necessário obter os extratos da planta e/ou óleos essenciais, e realizar uma caracterização fitoquímica. Os métodos de extração são aplicados ao cultivar partes mais ativas da planta como folhas, raízes, caules ou flores, visto que nestas partes estão ricamente mais presentes seus constituintes, usando solventes seletivos e procedimentos padrões ${ }^{17}$.

A amplitude das avaliações biológicas destes vegetais permite a aplicação de diferentes métodos para a constatação de um efeito farmacológico ${ }^{18}$. Com isso, torna-se interessante a avaliação antimicrobiana sobre biofilmes polimicrobianos envolvendo espécies fúngicas e bacterianas, posto que, naturalmente os micro-organismos se encontram distribuídos nos diferentes nichos ecológicos em associações interespecíficas ${ }^{18}$.

Dentre os micro-organismos de interesse, podemos citar Candida albicans, Staphylococcus aureus, Enterococcus faecalis, Streptococcus mutans e Pseudomonas aeruginosa que são de grande importância para Odontologia ${ }^{19}$, de modo que a ação de extratos de plantas sobre tais biofilmes pode ser bem relevante na prevenção e controle de diferentes infecções orais ${ }^{18}$.

Segundo o estudo realizado por Guimarães et al. ${ }^{10}$, sobre a atividade antimicrobiana in vitro do extrato aquoso e do óleo essencial do Alecrim, os resultados mostraram atividade inibitória em cepas de bactérias Gram-negativas sobre culturas avaliadas, corroborando com os achados na literatura a respeito da sua ação antibacteriana.

No estudo produzido por Fratini et al. ${ }^{20} \mathrm{o}$ óleo essencial de $R$. officinalis $L$. apresentou efeito antibacteriano para $S$. aureus e $S$. xylosus. Este produto vegetal promoveu inibição do crescimento das bactérias, demonstrado por teste de disco-difusão em ágar, onde foram gerados halos de inibição de 6,3 $\mathrm{mm}$ para $S$. aureus e de $8 \mathrm{~mm}$ para $S$. xylosus. Logo, $R$. officinalis $L$. apresentou efeitos como antimicrobianos.

A partir de uma pesquisa executada por Silva et al. ${ }^{21}$, foi possível observar a ação do extrato aquoso do $R$. officinalis $L$. na migração de neutrófilos do sangue ao tecido inflamado e produção de mediadores quimiotáticos e estresse oxidativo ocasionados por essa migração oriundo de um processo inflamatório. Com a aplicação do extrato ficou evidente a redução na migração de neutrófilos, bem como, diminuição dos níveis de mediadores inflamatórios no exsudato. Ainda, ficou comprovada in vitro a diminuição da atração de neutrófilos e da produção de NO. Com isso, os autores sugeriram a possibilidade de aplicação terapêutica do extrato de $R$. officinalis $L$. em casos de reações inflamatórias agudas.

Foi proposto ainda que o extrato etanólico de $R$. officinalis $L$. pode promover efeito protetor contra danos ao $\mathrm{DNA}^{22}$. Segundo a literatura, linfócitos humanos expostos ao extrato de $R$. officinalis $L$. não apresentaram genotoxicidade, no grupo tratado com o extrato e exposto ao peróxido de hidrogênio (H2O2), foi notado efeito protetor do $\mathrm{DNA}^{23}$ além de verificar-se seu efeito na migração de células imunes $^{21}$, assim, o $R$. officinalis $L$. apresenta efeitos como antitumoral ${ }^{24}$.

○Aplicações odontológicas do Rosmarinus officinalis $L$

Sob uma perspectiva Odontológica, em uma pesquisa efetuada por Bonilla et al. ${ }^{25}$ com objetivo de verificar o efeito do óleo essencial de Rosmarinus officinalis em comparação com o Metronidazol sobre o Porphyromonas gingivalis cultivado in vitro para combater a doença periodontal, os resultados indicaram que, embora o óleo essencial tenha atividade antimicrobiana ainda foi inferior à droga já existente. A diferença entre os resultados se deve ao fato de que avaliar compostos com possível atividade antimicrobiana, em concentrações desconhecidas e incorporado na matriz complexa do óleo essencial, torna-se complexo contra uma droga com um mecanismo conhecido de ação e atividade antimicrobiana amplamente estudado.

Outrossim, em estudos com bactérias causadoras da doença periodontal, como a Porphyromonas gingivalis, e geralmente eliminado por clorhexidina $0,12 \%$, Soto e Abigail $^{26}$ utilizaram o óleo essencial de Rosmarinus officinalis (Alecrim) e 
clorhexidina a $0,12 \%$ comparando seus efeitos sobre a bactéria como uma tentativa para encontrar um agente antimicrobiano natural, eficaz e econômico. Concluiu-se que o óleo essencial de Rosmarinus officinalis (Alecrim) teve um efeito inibidor contra Porphyromonas gingivalis porém inferior comparado a clorhexidina $0,12 \%$.

Em se tratando dos achados referentes às infeções fúngicas proposto por Gauch et al. ${ }^{27}$, o $R$. officinalis $L$. apresentou capacidade de interferir na filamentação in vitro de amostras clínicas de $C$. albicans, através de um importante fator de virulência desta levedura. A avaliação foi conduzida com $C$. albicans provenientes de próteses dentárias. Foi observado que a adição do óleo essencial de $R$. officinalis $L$. promoveu total inibição da filamentação das leveduras.

Solano et al. $^{28}$ propuseram um estudo que determinou-se a inibição do crescimento bacteriano in vitro de Streptococcus mutans, utilizando extratos aquoso e oleoso de Rosmarinus officinalis (Alecrim), aplicando o teste de disco-difusão. Cada um dos grupos teve um controle positivo de clorhexidina $0,12 \%$ e um controle negativo de agua destilada. $\mathrm{O}$ extrato aquoso de Alecrim não apresentou nenhum efeito antibacteriano sobre o $S$. mutans. Todavia, o extrato oleoso apresentou ação antibacteriano sobre S. mutans, sendo similar à clorhexidina $0,12 \%$.

Além disso, foi constatado também atividade antimicrobiana na pesquisa processada por Valones et al. ${ }^{29}$ cujo objetivo foi avaliar a atividade antimicrobiana de um dentifrício à base de extrato alcoólico de Alecrim sobre bactérias orais como Streptococcus mutans, Streptococcus oralis e Lactobacillus rhamnosus, comparando-o a um dentifrício herbal disponível no mercado. O dentifrício contendo extrato de alecrim foi capaz de inibir o crescimento de $S$. mutans, $S$. oralis e $L$. rhamnosus, revelando uma atividade antimicrobiana semelhante ao dentifrício disponível comercialmente na inibição de $S$. mutans e $S$. oralis.

Em uma pesquisa clínica randomizada feita por Tenorio ${ }^{30}$ também utilizando dentifrício à base do extrato alcoólico do alecrim sobre o biofilme bucal, comparando o extrato com um marca convencional (marca Sorrisoß) verificou ação antimicrobiana no extrato alcoólico do dentifrício. Após 30 dias de uso de cada dentifrício, todos os encontros foram registrados os seguintes parâmetros de cada indivíduo: Índice de Placa (IP) e Índice de Sangramento Gengival (ISG). Desta forma, o dentifrício fitoterápico obteve resultados semelhantes com o dentifrício convencional. No entanto, produziu uma diminuição maior no sangramento gengival, produzindo efeitos periodontais relevantes.

$R$. officinalis $L$. tem sido extensivamente estudado em relação à sua ação sobre microorganismos, contudo, seu efeito sobre microorganismos agrupados em biofilmes e associações polimicrobianas não foram avaliados ${ }^{31}$.

Estudo realizado por Oliveira et al. ${ }^{31}$ avaliou o efeito do extrato de alecrim sobre a viabilidade de biofilmes monomicrobianos de Candida albicans, Staphylococcus aureus, Enterococcus faecalis, Streptococcus mutans e Pseudomonas aeruginosa, bem como, sobre biofilmes polimicrobianos de $C$. albicans associada com $S$. aureus, E. faecalis, $S$. mutans ou $P$. aeruginosa. Biofilmes monomicrobianos de $C$. albicans, $S$. aureus, $S$. mutans e $P$. aeruginosa, foram afetados pelo extrato de alecrim, bem como, os biofilmes polimicrobianos de $C$. albicans associada com S. aureus, E. faecalis, $S$. mutans ou $P$. aeruginosa em biofilmes polimicrobianos, apresentando significativas reduções de viabilidade.

Diante disso, é possível encontrar na literatura estudos que relatam algumas atividades farmacológicas de $R$. officinalis, como efeito antimicrobiano $^{32}$, antibacteriano ${ }^{33}$, antifúngico ${ }^{27,15}$, antimicobacteriano $^{34}$, anti-inflamatório ${ }^{35,15}$, antioxidante ${ }^{36,37,32}, \quad$ antimutagênico ${ }^{38}$, neuroprotetivo ${ }^{39,40}$, cardioprotetor ${ }^{41}$, modulador de estresse oxidativo ${ }^{42,43}$ e DNA-protetivo ${ }^{44}$.

\section{CONCLUSÃO}

Diante disso, o efeito antimicrobiano produzido a partir de extratos do $R$. officinalis $L$. tem sido positivo segundo relatos da literatura, no entanto, mais estudos clínicos precisam ser realizados para enriquecer os atuais estudos, uma vez que sugere-se que a $R$. officinalis possua eficácia como planta fitoterápica no tratamento de diversas injúrias bucais por apresentar-se como um forte agente antimicrobiano.

\section{REFERÊNCIAS}

1. Severino VGP, Felixa MA, Silva MFGF, Lucarini R, Martins CHG. Chemical study of Hortia superba (Rutaceae) and investigation of the antimycobacterial activity of crude extracts and constituents isolated from Hortia species. Quím Nova. 2015;38:42-5.

2. Bardaji DK, Reis EB, Medeiros TC, Lucarini R, Crotti AE, Martins $\mathrm{CH}$. Antibacterial activity of commercially available plant-derived essential oils against oral pathogenic bacteria. Nat Prod Res. 2015;13:1-4.

3. Newman DJ, Cragg GM. Natural products as sources of new drugs from 1981 to 2014. J Nat Prod. 2016;79:629-61.

4. Suleimen E, Ibataev ZH, Iskakova $\mathrm{ZH}$, Ishmuratova M, Ross S, Martins CHG. Constituent composition and biological activity of essential oil from Artemisia terrae-albae. Chem Nat Compd. 2016;52:173-75.

5. Jardak M, Elloumi-Mseddi J, Aifa S, Mnif S. Chemical composition, anti-biofilm activity and 
potential cytotoxic effect on cancer cells of Rosmarinus officinalis L. essential oil from Tunisia. Lipids Health Dis. 2017;16(1):190.

6. Habtemariam S. The therapeutic potential of rosemary (Rosmarinus officinalis) diterpenes for Alzheimer's disease. Evid Based Complement Alternat Med. 2016,2680409.

7. Ferreira-Filho JCC, Gondim BLC, Cunha DA, Figueiredo CC, Valença AMG. Physical properties and antibacterial activity of herbal tinctures of Calendula (Calendula officinalis L.) and Cashew Tree (Anacardium occidentale L.). Pesqui Bras Odontopediatria Clin Integr.2014; 14(1):49-53.

8. Raskovic A, Milanovic I, Pavlovic N, Cebovic T, Vukmirovic S, Mikov M. Antioxidant activity of rosemary (Rosmarinus officinalis L.) essential oil and its hepatoprotective potential. BMC Complement Altern Med. 2014.14:225.

9. Moura AR, Medeiros IC, Souza MJMF. Avaliação do teor de óleo essencial de rosmarinus sp. cultivado em dois níveis de luminosidade. REFACER. 2016;5(2).

10. Guimarães CC, Ferreira TC, Oliveira RCF, Simioni PU, Ugrinovich LA. Atividade antimicrobiana in vitro do extrato aquoso e do óleo essencial do alecrim (Rosmarinus officinalis L.) e do cravo-da-índia (Caryophyllus aromaticus L.) frente a cepas de Staphylococcus aureus e Escherichia coli. R bras Bioci. 2017;15(2):83-9.

11. Bernardo WM, Nobre MRC, Jatene FB. A prática clinica baseada em evidências. Parte II: buscando as evidências em fontes de informação. Rev Assoc Med Bras. 2004;50(1):1-9.

12. Hotwani K, Baliga S, Sharma K. Phytodentistry: use of medicinal plants. J Complement Integr Med 2014;11:233-251.

13. Aumeeruddy-Elalfi Z, Gurib-Fakim A, Mahomoodally MF. Chemical composition, antimicrobial and antibiotic potentiating activity of essential oils from 10 tropical medicinal plants from Mauritius. J Herb Med. 2016;6(2):88-95.

14. Aumeeruddy-Elalfi Z, Gurib-Fakim A, Mahomoodally F. Antimicrobial, antibiotic potentiating activity and phytochemical profile of essential oils from exotic and endemic medicinal plants of Mauritius. Ind Crops Prod. 2015; 71:197-204.

15. Silva BN, Nakassugi LP, Faggion POJ, Kohiyama CY, Mossini SA, Grespan R, et al. Antifungal activity and inhibition of fumonisin production by Rosmarinus officinalis L. essential oil in Fusarium verticillioides (Sacc.) Nirenberg. Food Chem.2015;166: 330-36.

16. Vanin AB. Produção, propriedades biológicas, antioxidantes e toxicidade do bioaromatizante obtido via esterificação enzimática de óleo essencial do cravo-da-índia (Caryophyllus aromaticus) [tese]. Erechim: Universidade Regional Integrada do Alto Uruguai e das Missões; 2014.

17. Badal-McCreath S, Delgoda R. Pharmacognosy: fundamentals, applications and strategies. Academic Press, London, UK, 2016.

18. Oliveira JR. Avaliação de atividades biológicas dos extratos de rosmarinus officinalis 1. (alecrim) e thymus vulgaris 1. (tomilho) [tese]. São José dos Campos: Instituto de Ciência e Tecnologia, UNESP - Univ Estadual Paulista; 2016.

19. Oliveira JR, Aguiar-Almeida RB, Vilela PGF, Oliveira FE, Rocha RF, Jorge AO, et al. Control of microorganisms of oral health interest with Arctium lappa L. (burdock) extract non-cytotoxic to cell culture of macrophages (RAW 264.7). Arch Oral Biol. 2014;59:808-14.

20. Fratini F, Casella S, Leonardi M, Pisseri F, Ebani VV, Pistelli L, et al. Antibacterial activity of essential oils, their blends and mixtures of their main constituents against some strains supporting livestock mastitis. Fitoterapia. 2014;96:1-7.

21. Silva AM, Machado ID, Santin JR, Melo IL, Pedrosa GV, Genovese MI et al. Aqueous extract of Rosmarinus officinalis L. inhibits neutrophil influx and cytokine secretion. Phytother Res 2015;29:125-33.

22. Razavi-Azarkhiavi K, Behravan J, Mosaffa F, Sehatbakhsh S, Shirani K, Karimi G. Protective effects of aqueous and ethanol extracts of rosemary on $\mathrm{H}_{2} \mathrm{O}_{2}$-induced oxidative DNA damage in human lymphocytes by comet assay. $\mathrm{J}$ Complement Integr Med. 2014;11:27-33.

23. Oliveira JF, Jesus D, Figueira LW, Oliveira FE, Soares CP, Camargo SEA, et al. Biological activities of Rosmarinus officinalis L. (rosemary) extract as analyzed in microorganisms and cells. Exp Biol Med (Maywood). 2017;242(6):625-34.

24. González-Vallinas M, Molina S, Vicente G, Zarza V, Martín-Hernández R, García-Risco MR, Fornari T, Reglero G, Ramírez de Molina A. Expression of microRNA-15b and the glycosyltransferase GCNT3 correlates with antitumor efficacy of Rosemary diterpenes in colon and pancreatic cancer. PLoS One. 2014;9(6):e98556.

25. Bonilla D.M, Mendoza Y, Moncada C.E, Murcia O, Rojas Á.P, Callen J, et al. Efecto del aceite esencial de Rosmarinus officinalis sobre Porphyromonas gingivalis cultivada in vitro, Rev Colomb Cienc Quím Farm. 2016;45(2):275-87.

26. Soto C, Abigail Y. Efecto inhibidor del aceite esencial de rosmarinus officinalis (romero) y clorhexidina al $0,12 \%$ sobre porphyromonas gingivalis. estudio in vitro [tese]. Lima: Universidad Privada Norbert Wiener Facultad de Ciencias de la Salud Escuela Académico Profesional de Odontología; 2015. 
27. Gauch LM, Silveira-Gomes F, Esteves RA, Pedrosa SS, Gurgel ES, Arruda AC, et al. Effects of Rosmarinus officinalis essential oil on germ tube formation by Candida albicans isolated from denture wearers. Rev Soc Bras Med Trop. 2014;47(3):389-91.

28. Solano XK, Silva TJM, Gutiérrez MIZ. Inhibición del Streptococcus mutans, mediante el uso de extracto acuoso y oleoso de Rosmarinus officinalis "romero". Odontología. 2016;19(2):29-34.

29. Valones MAA, Higino JS, Souza PRE, Crovella S, Júnior AFC, Carvalho AAT. Dentifrice containing extract of Rosmarinus officinalis Linn.: an antimicrobial evaluation. Braz Dent J. 2016; 27(5):497-501.

30. Tenorio LA. a avaliação clínica da atividade do dentifrício à base do extrato de rosmarinus officinalis linn. (alecrim) sobre o biofilme dentalum estudo preliminar [dissertação]. Recife: Programa de Pós-Graduação em Odontologia do Centro de Ciências da Saúde da Universidade Federal de Pernambuco (UFPE); 2014.

31. Oliveira JR, Jesus D, Oliveira LD. Rosmarinus officinalis 1. (Rosemary) extract decreases the biofilms viability of oral health interest. Braz Dent Sci 2017;20(1):64-9.

32. Guerra-Boone L, Alvarez-Roman R, SalazarAranda R, Torres-Cirio A, Rivas-Galindo VM, Torres NW, et al. Antimicrobial and antioxidant activities and chemical characterization of essential oils of Thymus vulgaris, Rosmarinus officinalis and Origanummajorana from northeastern Mexico. Pak J Pharm Sci. 2015;28(1):363-69.

33. Irshaid FI, Tarawneh KA, Jacob JH, Alshdefat AM. Phenol content, antioxidant capacity and antibacterial activity of methanolic extracts derived from four Jordanian medicinal plants. Pak J Biol Sci. 2014;17(3):372-79.

34. Abuzeid N, Kalsum S, Koshy RJ, Larsson M, Glader M, Andersson H, et al. Antimycobacterial activity of selected medicinal plants traditionally used in Sudan to treat infectious diseases. J Ethnopharmacol. 2014;157:134-39.

35. Rocha J, Eduardo-Figueira M, Barateiro A, Fernandes A, Brites D, Bronze R, et al. Antiinflammatory effect of rosmarinic acid and an extract of Rosmarinus officinalis in rat models of local and systemic inflammation. Basic Clin Pharmacol Toxicol. 2015;116(5):398-413.

36. Motlagh MK, Sharafi M, Zhandi M, MohammadiSangcheshmeh A, Shakeri M, Soleimani M et al. Antioxidant effect of rosemary (Rosmarinus officinalis L.) extract in 11 soybean lecithin-based semen extender following freeze-thawing process of ram sperm. Cryobiology. 2014;69(2):217-22.

37. Dias LS, Menis ME, Jorge N. Effect of rosemary (Rosmarinus officinalis) extracts on the oxidative stability and sensory acceptability of soybean oil. J Sci Food Agric. 2015;95(10):2021-7.

38. Felicidade I, Lima JD, Pesarini JR, Monreal AC, Mantovani MS, Ribeiro LR, et al. Mutagenic and antimutagenic effects of aqueous extract of rosemary (Rosmarinus officinalis L.) on meristematic cells of Allium cepa. Genet Mol Res. 2014;13(4):9986-96.

39. Lin CY, Chen JH, Fu RH, Tsai CW. Induction of $\mathrm{Pi}$ form of glutathione S-transferase by carnosic acid is mediated through PI3K/Akt/NF- $\mathrm{BB}$ pathway and protects against neurotoxicity. Chem Res Toxicol. 2014;27(11):1958-66.

40. Wu CR, Tsai CW, Chang SW, Lin CY, Huang LC, Tsai CW. Carnosic acid protects against 6hydroxydopamine-induced neurotoxicity in in vivo and in vitro model of Parkinson's disease: Involvement of antioxidative enzymes induction. Chem Biol Interact. 2015;225:40-6.

41. Li XL, Liu JX, Li P, Zheng YQ. Protective effect of rosmarinic acid onhypoxia/reoxygenation injury in cardiomyocytes. Zhongguo Zhong Yao Za Zhi. 2014;39(10):1897-901.

42. El-Demerdash FM, Abbady EA, Baghdadi HH. Oxidative stress modulation by Rosmarinus officinalis in creosote-induced hepatotoxicity. Environ Toxicol. 2016;31(1):85-92.

43. Sebai H, Selmi S, Rtibi K, Gharbi N, Sakly M. Protective effect of Lavandula stoechas and Rosmarinus officinalis essential oils against reproductive damage and oxidative stress in alloxan-induced diabetic rats. J Med Food. 2015;18(2):241-9.

44. Horvathova E, Navarova J, Galova E, Sevcovicova A, Chodakova L, Snahnicanova Z, et al. Assessment of antioxidative, chelating, and DNA-protective effects of selected essential oil components (eugenol, carvacrol, thymol, borneol, eucalyptol) of plants and intact Rosmarinus officinalis oil. J Agric Food Chem. 2014;62(28):6632-9. 
http://dx.doi.org/10.21270/archi.v10il.3197

\section{CONFLITO DE INTERESSES}

Os autores declaram não haver conflitos de interesse.

\section{AUTOR PARA CORRESPONDENNCIA}

\section{Mírian Alencar de Medeiros}

Rua João Cabloco, número 34, bairro Centro,

5931000 Ipueiro - RN

Telefone: (83) 99937-3179.

E-mail: mamedeiros2011@live.com

Submetido em 15/08/2018

Aceito em 04/10/2018 